Full Paper

\title{
Isolation and Characterization of a Novel Four-Transmembrane Protein PMP22CD Specifically Expressed in the Testis
}

\author{
Minghua Wang ${ }^{1}$, Guanmei Luo ${ }^{2}$, Fang $\mathrm{Li}^{3}$, Shiliang $\mathrm{Wu}^{1}{ }^{1}$, Juxiang Jiang ${ }^{1}$ and Chaoqun \\ Huang 1,*
}

1 Department of Biochemistry and Molecular Biology, Institute of Biochemical Engineer, Medical School, Soochow University, Suzhou, P.R. China

2 School of Medical Sciences, University of Birmingham, Edgbaston, Birmingham B15 2TT, UK

3 Department of Histology and Embryology, Medical School, Soochow University, Suzhou, P.R.

China

* Author to whom correspondence should be addressed. Tel: +86-512-65880108; Fax: +86-51265880103; Email: chaoqunhuang89@yahoo.com

Received: 26 September 2006 / Accepted: 18 October 2006 / Published: 25 October 2006

\begin{abstract}
PMP22_Claudin family proteins play important roles in cell tight junction. In this study, we have identified a novel member of this family, PMP22CD. Human PMP22CD was first discovered by database sequence mining and analysis, and verified by cloning and sequencing. PMP22CD was isolated from the human testis cDNA library and mapped to chromosome 11q24.1 by browsing the UCSC genomic database. It contains an ORF with a length of $675 \mathrm{bp}$, encoding a protein that contains a putative PMP22_Claudin domain with four transmembrane helices. Its molecular weight and isoelectric point are predicted to be $25.8 \mathrm{kDa}$ and 8.42 , respectively. The PMP22CD protein is highly conservative in mammal animals. Phylogenetic tree analysis indicated that PMP22CD stands for a new subgroup in the PMP22/EMP/Claudin family. RT-PCR analysis showed that PMP22CD was specifically expressed in the testis. Green fluorescence protein localization analysis showed that PMP22CD mainly surrounded the nuclear membrane, with a minority distribution in the cytoplasm. These results suggested that PMP22CD is a distant member of the PMP22/EMP/Claudin family and that it may have a novel function that does not involve cell tight junction because it is not located at the cell membrane.
\end{abstract}

Keywords: PMP22_Claudin; Expression pattern; Testis; GFP localization 


\section{Introduction}

Two types of cell junction, Tight and adherent junctions, play roles in maintaining cell-to-cell adhesion[1]. Tight junction (TJ) is very important for barrier function in epithelial and endothelial cells. TJ are composed of proteins with four transmembrane helices. These proteins have low sequence similarity but do have a structural homology to the tetraspan membrane protein family members. These proteins include Occludin[2], PMP22 (Peripheral Myelin Protein 22) [3], EMP (Epithelial Membrane Protein) and Claudin family members[4-6]. Recently, a number of claudin family membrane proteins related to TJ have been identified. So far, the claudin family has expanded to more than 20 members[7]. In addition, two distantly related members of the claudin family, BCMP1 (brain cell membrane protein 1) [8] and CLP24 (claudin like protein of $24 \mathrm{kDa}$ ) [9], were identified. All claudins encode 20-27 kDa proteins with lengths of 207-264 amino acids. Claudins contain four transmembrane domains and two extracellular loops. The first extracellular loop is significantly longer than the second one, and a short intracellular tail is in its c-terminus. The last few amino acids of this tail are highly conserved within the family[10], and they usually show the PDZ (protein-protein interaction domain) binding motif YV or ФXФ ( $\Phi$ stands for hydrophobic amino acid, X stand for any amino acid) [10].

Despite the fact that the complete nucleotide sequence of the human genome is known, many genes encoding membrane proteins were continuously discovered, So far some membrane proteins still remain unidentified. We are interested in searching for other integral membrane proteins of TJ. Currently it is still a valid method to identify membrane protein encoding genes in human genomes using a method of in silico analysis combined with experimental cloning[11, 12]. In this study, we have identified and characterized a novel human gene PMP22CD (PMP22 claudin domain containing) by using EST database sequences as a departure point and confirmed by experimental cloning. The PMP22CD gene contains an ORF with a length of $675 \mathrm{bp}$, encoding a protein with putative PMP22_Claudin domain. The PMP22CD protein is composed of four transmembrane helices and it is a distantly related member of the PMP22/EMP/Claudin families.

The sequence data reported here have been submitted to the GenBank database under accession number AY634366.

\section{Materials and Methods}

\subsection{Cloning of the gene PMP22CD}

EST database (http://ncbi.nlm.nih.gov) [13] searches allowed us to identify a cluster of ESTs(Accession No. AA423960, AA437068, AA625777, AA629054, AA629312, BX091037, BX106484) which did not show any homology with known genes, Identified ESTs were assembled into a contig using Vector NTI package (Informax Co, Ltd.). To verify the sequence of the contig, primers of PMP22CD-up (5' - ACTCACAATGGTGCATGTTTC-3' corresponding to nucleotides 181201nt) and PMP22CD-down (5'-TAGATTGCCAAATCACAGAGC-3' corresponding to nucleotides 877-897nt) were designed and synthesized (Biocolour BioTech). PCR was performed with PMP22CD primers, human testis cDNA library (Clontech Co, Ltd.) used as template. The PCR conditions were as follows: $94^{\circ} \mathrm{C}$ for $5 \mathrm{~min}, 35$ cycle of $20 \mathrm{~s}$ at $94^{\circ} \mathrm{C}, 30 \mathrm{~s}$ at $56.5^{\circ} \mathrm{C}, 50 \mathrm{~s}$ at $72^{\circ} \mathrm{C}$, followed by a final 
extension of $5 \mathrm{~min}$ at $72^{\circ} \mathrm{C}$. The PCR product was subjected to T-A cloning and was sequenced on an ABI PRISM sequencer.

\subsection{In silico analysis}

To determine the mapping information, the cDNA sequence of PMP22CD was applied for genomic searching at http://genome.ucsc.edu. BLASTP tool (http://www.ncbi.nlm.nih.gov) was applied for identifing the orthologs of PMP22CD in different species. The SMART tool was used for domain searching (http://smart.embl-heidelbeg.de), and the Vector NTI package (Informax Co, Ltd.) for protein molecular weight prediction. PSORT (http://psort.nibb.ac.jp) was used for subcellular localization prediction. The HMMTOP server (http://www.enzim.hu/hmmtop) was used for the prediction of the transmembrane helices and topology[14]. GeneDoc software was used for sequence alignment. Phylogenetic tree analysis of the amino acid sequences of PMP22CD, members of PMP22_claudin family and EMP was performed using the ClustalW program at http://www.ebi.ac.uk/clustalw/. The GenBank accession numbers used for analysis are: PMP22CD (human NP_001013765, monkey XP_001108697, dog XP_852701, mouse BAB24641, rat NP_001019530, cattle AAI09976); human claudins (claudin1 NP_066924, claudin2 NP_065117, claudin3 NP_001297, claudin4 NP_001296, claudin5 NP_003268, claudin6 NP_067018, Claudin7 NP_001298, Claudin8 NP_955360, Claudin9 NP_066192, Claudin10 NP_008915, Claudin11 NP_005593, Claudin12 NP_036261, Claudin14 NP_036262, Claudin15 NP_055158, Claudin16 NP_006571, Claudin17 NP_036263, Claudin18 NP_001002026, Claudin19 NP_683763, Claudin23 NP_919260); human PMP22 NP_696996, EMP1 NP_001414, EMP2 NP_001415, EMP3 NP_001416, CLP24 AAT78423, BCMP1 NP_113630.

\subsection{Expression pattern analysis of PMP22CD}

The expression pattern of $P M P 22 C D$ was determined by PCR amplification using a cDNA panel of 14 human tissues (lung, placenta, prostate, liver, heart, brain, stomach, uterus, testis, skeletal muscle, colon, kidney, bladder and ovary), purchased from Clontech Institute, Inc. The primer pair of PMP22CD-RT-A (5'- TATGATGACGTCGAGCCTTGGC -3' corresponding to nucleotides 412433nt) and PMP22CD-RT-B (5'- GTGCACGGACAATGCTACGAGG -3' corresponding to nucleotides 806-827nt) was used, the length of the PCR product is $416 \mathrm{bp}$. The expression of the $\beta-M G$ was analyzed as a control: sense prime (5'-CTCGTGCTACTCTCTCTTTC-3'), antisense prime (5'CATGTCTCGATCCCACTTAAC-3'). The PCR conditions were the same as for cloning except that the prolongation period was changed to $30 \mathrm{~s}$.

\subsection{Analysis of subcellular localization}

The PCR product of the complete PMP22CD ORF with Hind III and Pst I restriction sites was subcloned into the Hind III/Pst I sites of pEGFP-C1 (Clontech), and produced the recombinant green fluorescence expression vector pEGFP-PMP22CD. Restriction sites had been introduced by the forward (5'-CGCAAGCTTCAATGGTGCATGTTTC-3') and reverse (5'GATCTGCAGATCACAGAGCCC AG-3') primers. COS7 cells were grown in Dulbecco's modified 
Eagle's medium supplemented with $10 \%$ fetal bovine serum (Gibco-BRL) and $1 \%$ penicillin/streptomycin at $37^{\circ} \mathrm{C}$ in a $5 \%$ humidified $\mathrm{CO}_{2}$ atmosphere. Cells were cultured directly on glass coverslips in six-well plates at a density of approximately $2 \times 10^{5}$ cells per well and incubated for $24 \mathrm{~h}$. The incubation was continued for another $3 \mathrm{~h}$ in the presence of the Superfect Reagent (Qiagen, Germany), $1.7 \mu \mathrm{g}$ pEGFP-PMP22CD, and pEGFP-C1 green fluorescence protein expression vector as a control. The medium was replaced by $10 \%$ charcoal-treated fetal calf serum (DCC, Hyclone). The cells were incubated for $48 \mathrm{~h}$ and then washed three times with PBS ( $\mathrm{pH} 7.4)$. The COS7 cells were fixed in 3\% paraformaldehyde for $20 \mathrm{~min}$ and then permeabilized with $0.5 \%$ Triton X-100 in PBS for 5 min[15]. After rinsing with PBS, DAPI (4',6-diamidino-2-phenylindole; $2.5 \mu \mathrm{g} / \mathrm{ml}$ ) (Invitrogen) in PBS was used to stain the nucleus for $30 \mathrm{~min}$. and visualized with a microscope equipped with fluorescence optics (Leica DM R2, Germany).

\subsection{Cell transfection and western blotting}

The pEGFP-PMP22CD fusion construct was transfected into COS7 cells. pEGFP-C1 was used as a control. The cells were harvested $48 \mathrm{~h}$ after transfection and washed twice with ice-cold phosphatebuffered saline (PBS), and lysed in SDS sample buffer. Proteins separated via SDS-PAGE were transferred onto a PVDF membrane using the semi-dry blotting method, and detected using the rabbit serum anti-GFP polyclonal antibody (Rockland).

\section{Results}

\subsection{Human PMP22CD gene and its genomic organization}

Seven ESTs (Accession No. AA423960, AA437068, AA625777, AA629054, AA629312, BX091037, BX106484) were assembled into a contig with a length of 1098bp as shown in Figure 1. In order to clone the PMP22CD gene by PCR, we first analyzed the tissue distribution of the ESTs from $P M P 22 C D$. We found that all ESTs were transcripted from the testis, so the human testis cDNA library was used as a template for PCR amplification. The band at expected size was excised from the agarose gel and cloned into pMD18-T vector $(\mathrm{TaKaRa})$ and subjected to sequencing. The sequencing result was consistent with the contig. The gene was named PMP22CD (PMP22 claudin domain containing protein), and submitted to GenBank with the GenBank Accession No. AY634366. PMP22CD cDNA is 1098bp in length, encoding a protein with 225 amino acids (Figure 2). The molecular weight and isoelectric point are predicted to be $25.8 \mathrm{kDa}$ and 8.42 , respectively, using Vector NTI software. It is composed of four exons and three introns (Table 1), the open reading frame is from 209 nucleotides to 883 nucleotides, the ATG start codon (nucleotides209-211) is preceded by an in-frame stop codon TAG, and there is a typical polyadenylation signal AATAAA. 


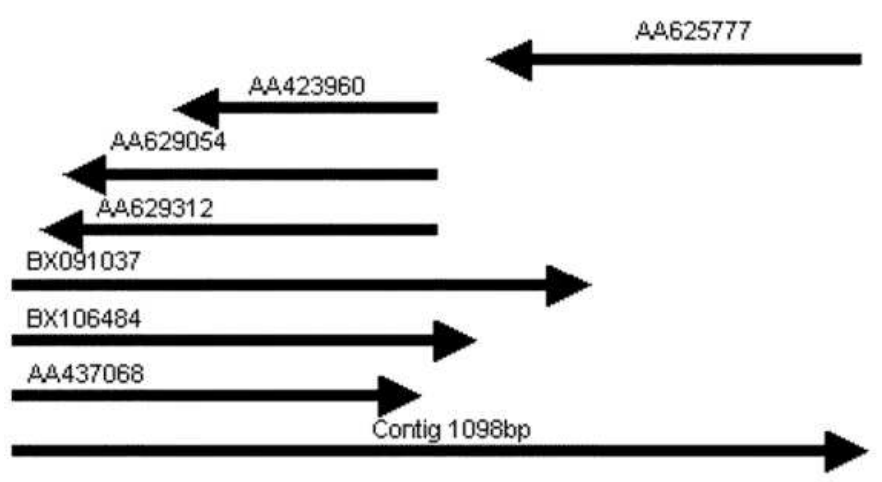

Figure 1. The assembled contig of PMP22CD. Seven ESTs (Accession No. AA423960, AA437068, AA625777, AA629054, AA629312, BX091037, BX106484) were used.

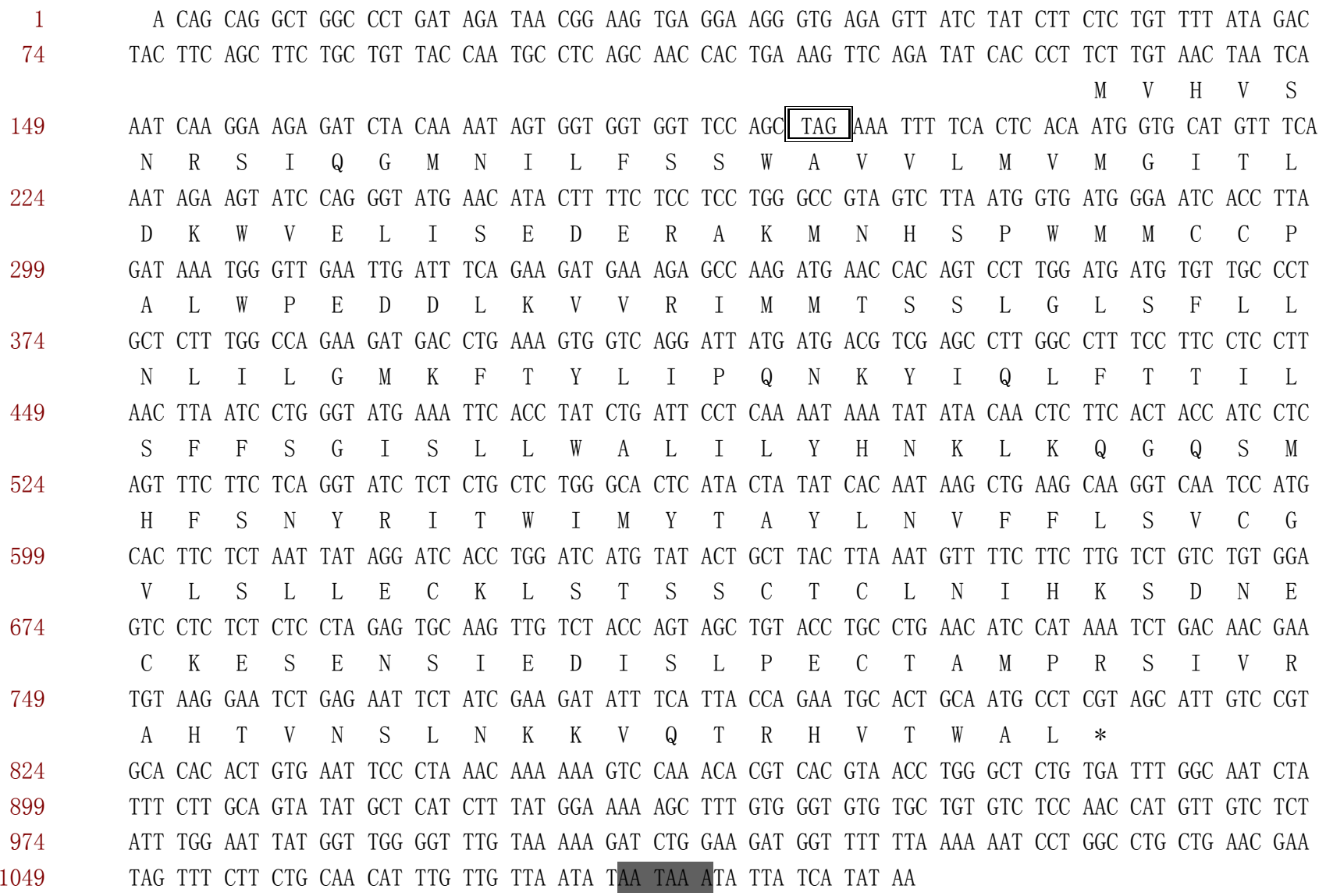

Figure 2. Nucleotide sequence and deduced protein sequence of the human $P M P 22 C D$ gene. $P M P 22 C D$ encodes a polypeptide of 225 amino acids, amino acids are identified by their one-letter code, nucleotides are numbered at the left side of each line. *, The termination codon TGA; AA TAA A, Polyadenylation signals; TAG, in-frame stop codon. 
Table 1. Genomic structure of $P M P 22 C D$ gene.

\begin{tabular}{llclcc}
\hline 3'Splice acceptor & $\begin{array}{l}\text { Exo } \\
\mathrm{n}\end{array}$ & $\begin{array}{l}\text { Size } \\
(\mathrm{bp})\end{array}$ & 5' Splice donor & Intron & $\begin{array}{l}\text { Size } \\
(\mathrm{bp})\end{array}$ \\
\hline cDNA end & 1 & 389 & TGGCCAGAAGgtcagaactg & 1 & 608 \\
ACAGCAGGCT & & & & & \\
tttccaacagATGACCTGAA & 2 & 147 & TTCTTCTCAGgtaacttcct & 2 & 280 \\
tggacatcagGTATCTCTCT & 3 & 135 & TCTGTCTGTGgtgagtgtct & 3 & 722 \\
tttccctagGAGTCCTCTC & 4 & 427 & TAATAAATATTATCATATAA & cDNA end
\end{tabular}

Exon sizes are given in bp and the exonic and intronic sequences at the splice junction are shown in capital and lowercase letters, respectively. The exon-intron splicing signals $\boldsymbol{g} \boldsymbol{t}$ and $\boldsymbol{a g}$ are in bold.

\subsection{In silico analysis of PMP22CD gene}

PMP22CD was mapped to chromosome $11 \mathrm{q} 24.1$ by browsing the UCSC genomic database (http://genome.ucsc.edu). Another interesting finding was that the gene PMP22CD and twelve olfactory receptor genes (OR6X1, OR6M1, OR6M2P, OR6M3P, OR8D4, OR4D5, OR6T1, OR10S1, OR10G4, OR10G9, OR10G7, OR10G8) are located close to each other and cluster together in the same chromosome loci 11q24.1 in the human (Figure 3).

The use of putative transmembrane domain prediction software (HMMTOP) revealed that the PMP22CD protein is composed of four alpha-helical transmembrane domains (11-30, 68-86, 101-120, 137-160) (Figure 4). The HMMTOP analysis also revealed that the N-terminus is outside of the membrane, the first loop of PMP22CD is longer than the second one, and that PMP22CD contains a very short $\mathrm{N}$-terminus tail, but a more than 65 residues long c-terminus.

A BLASTP search of published protein databases of NCBI for sequences similar to that of PMP22CD indicated that it had orthologs in monkey, dog, mouse, rat and cattle. Multiple alignment analysis was made by Gendoc software. The result in Figure 5a showed that PMP22CD was highly conservative in different species: there were six serine residues and ten leucine residues highly conserved in all the species, and five amino acid VTWAL was highly conserved in its c-terminus. Human PMP22CD shares a high degree of homology with the orthologs in monkey (82\% identity and $89 \%$ similarity), dog (53\% identity and $72 \%$ similarity), mouse (44\% identity and $67 \%$ similarity), rat (42\% identity and 68\% similarity), and cattle (41\% identity and 63\% similarity).

A multiple alignment analysis showed that PMP22CD has a very low homology with PMP22/EMP/Claudin family members (data not shown here). Although the amino acid sequence of PMP22CD share very low homology with the PMP22/EMP/Claudin family, the domain prediction result of searching in the SMART database showed that human PMP22CD protein contains a PMP22_Claudin domain from position 7 to position 158 (http://smart.embl-heidelbeg.de), and the Evalue is $0.011(<0.05)$.

Furthermore, we analyzed the evolutionary relationship among PMP22CD, different members of the PMP22/EMP/Claudin family, BCMP1 and CLP24, with bootstrap N-J tree analysis (Figure 5b. ), which shows that PMP22CD is one of the protein conserved during evolution. In addition, PMP22CD, 
the distant members of the Claudin family BCMP1 and CLP24, Claudin12 and claudin16 cluster together in the evolution tree.

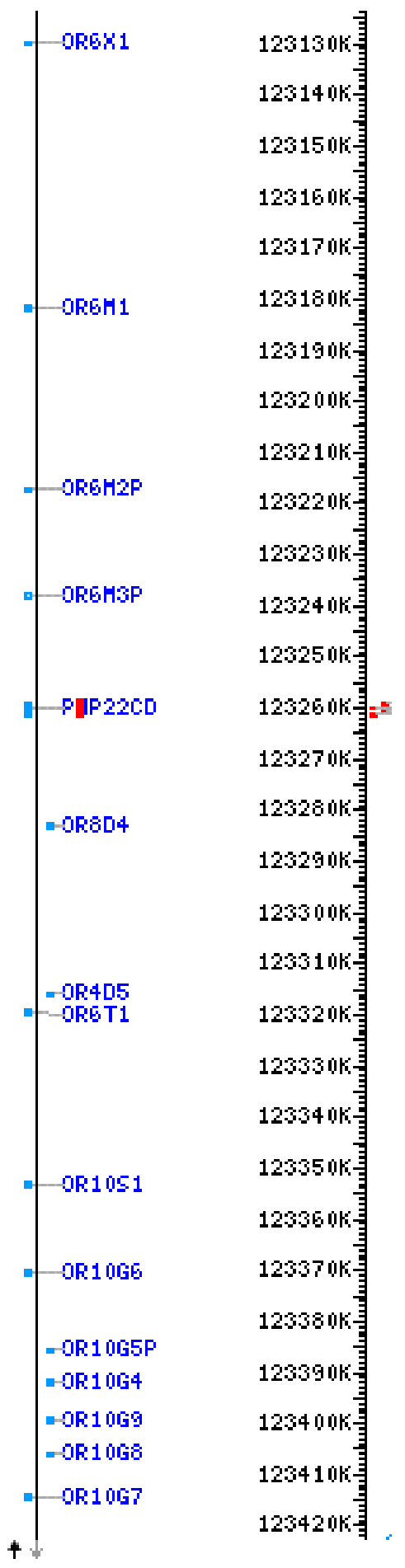

Figure 3. Genome location of PMP22CD and twelve olfactory receptor genes (OR6X1, OR6M1, OR6M2P, OR6M3P, OR8D4, OR4D5, OR6T1, OR10S1, OR10G4, OR10G9, OR10G7, OR10G8) in human chromosome. 


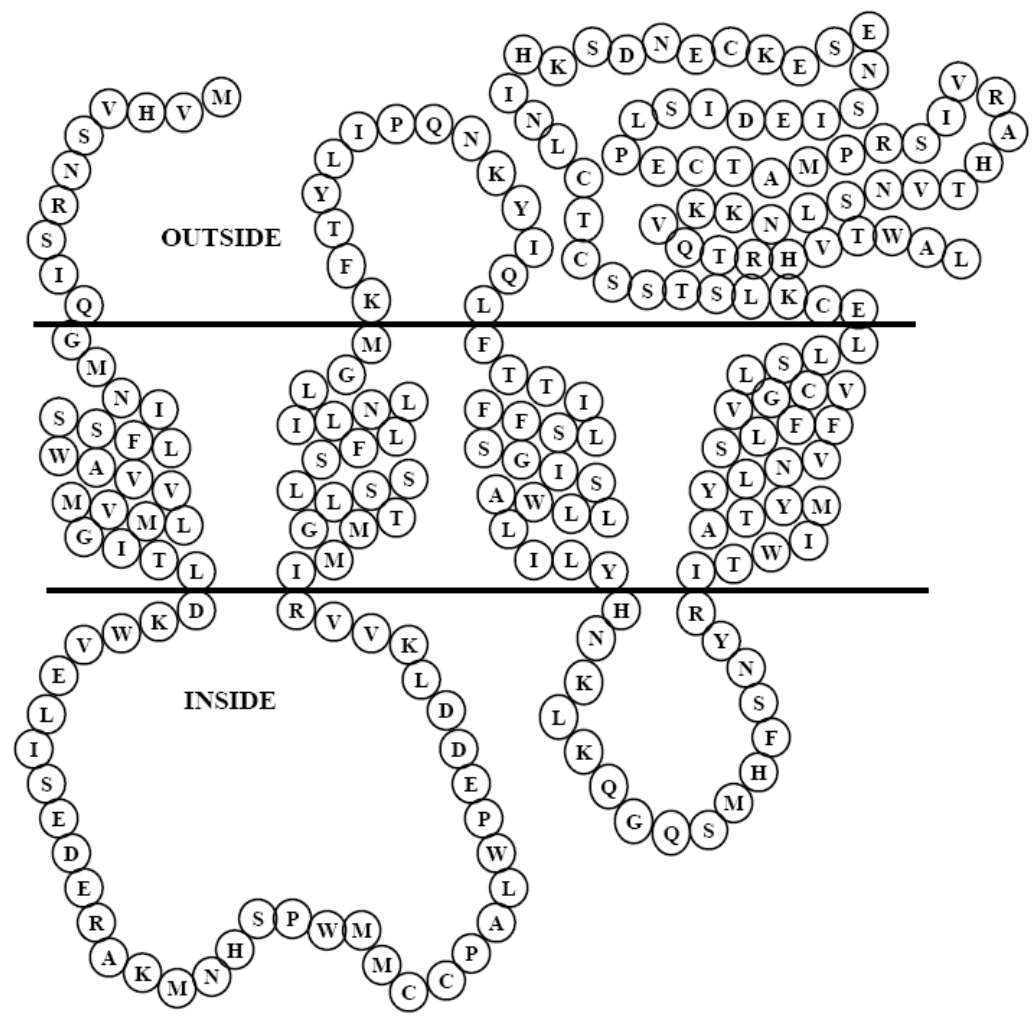

Figure 4. Predicted structure of PMP22CD in the membrane. The structure was drawn based on the prediction of HMMTOP. PMP22CD show four transmembrane domains and a long c-terminal domain.

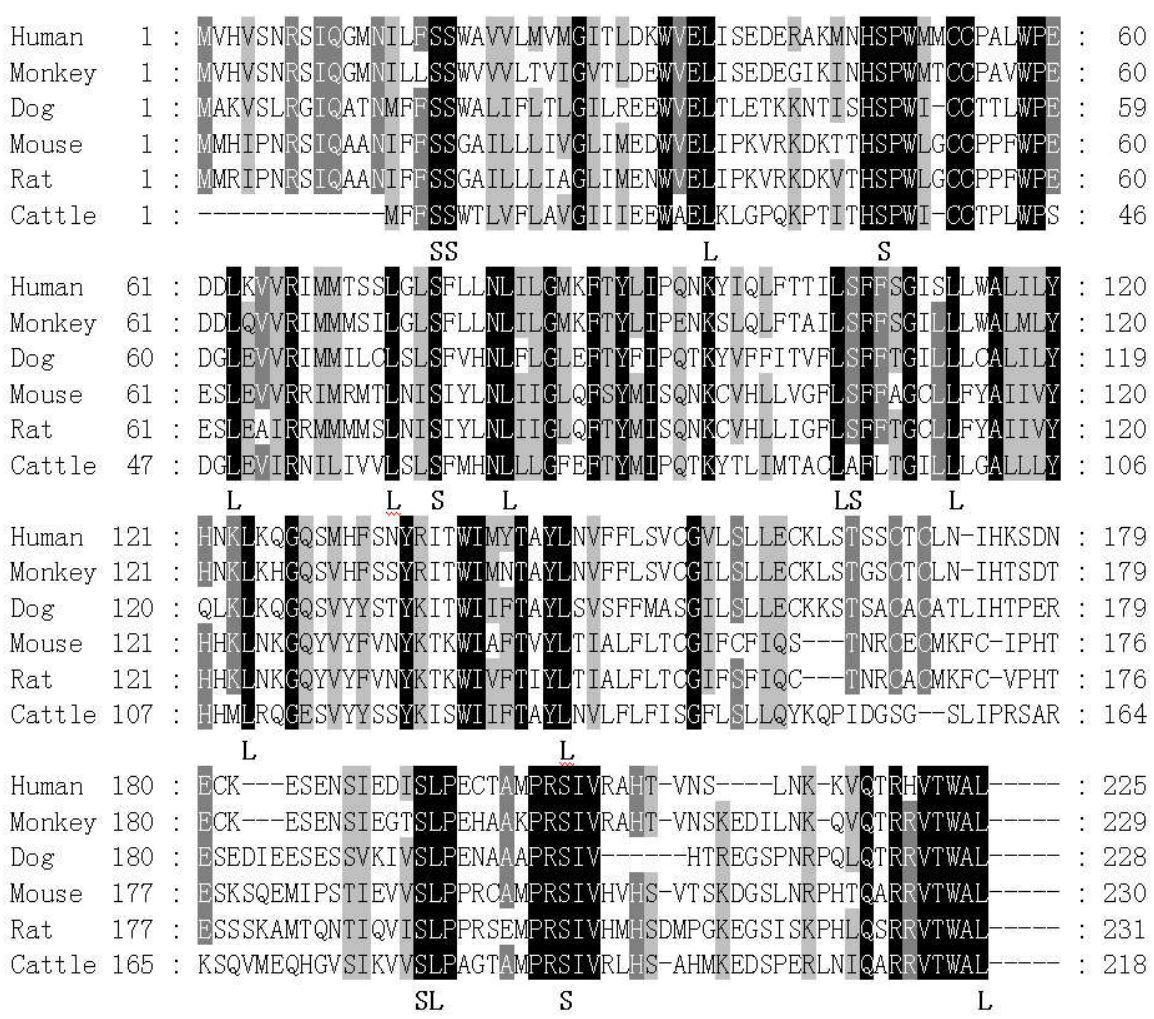

Figure 5a. The sequence alignment of human PMP22CD protein and its orthologs. Identical amino acids are shaded in black, similar amino acids are shaded in gray. Highly conservative serine and leucine were indicated The sequences used for alignment include: human (NP_001013765), monkey (XP_001108697), cattle (AAI09976), mouse (BAB24641), rat (NP_001019530), and dog (XP_852701). 


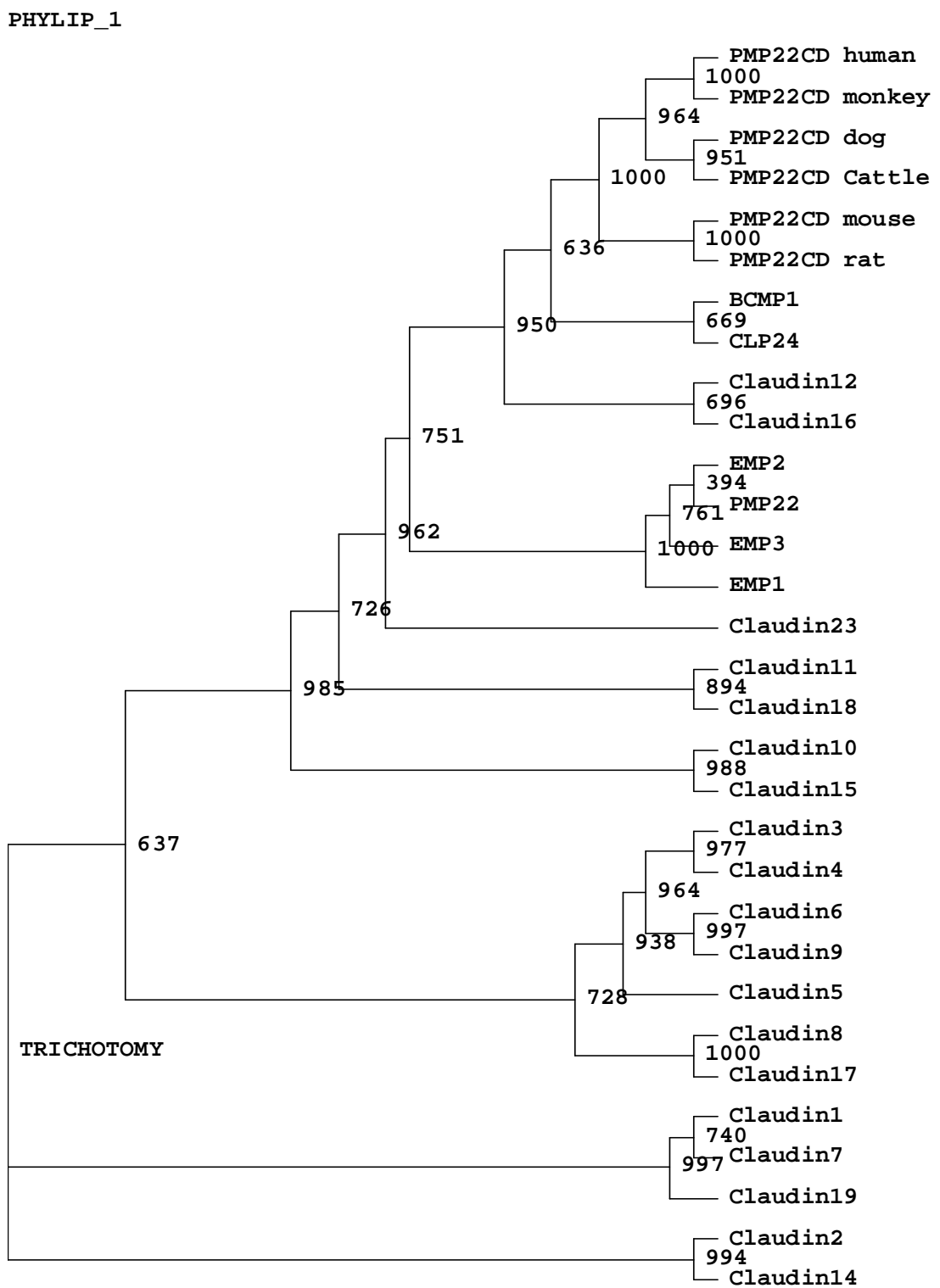

Figure 5b. Phylogenetic tree of PMP22CD and PMP22/EMP/Claudin family proteins. A phylogenetic tree was constructed with the bootstrap N-J method using program PHYLIP with 1000 bootstrap trials.

The number is the value that this cluster was found in 1000 trials.

\subsection{Expression pattern of PMP22CD gene}

Northern blotting and RT-PCR were used to determine the expression pattern of the gene. In order to investigate the tissue distribution of $P M P 22 C D$, a cDNA panel of 14 human tissues was used for RT-PCR analysis. The tissue distribution pattern of PMP22CD mRNA was shown in Figure 6 . The band was detected in testis, but no band was detected in the other tissues, this suggested that $P M P 22 C D$ was specifically expressed in testis. 


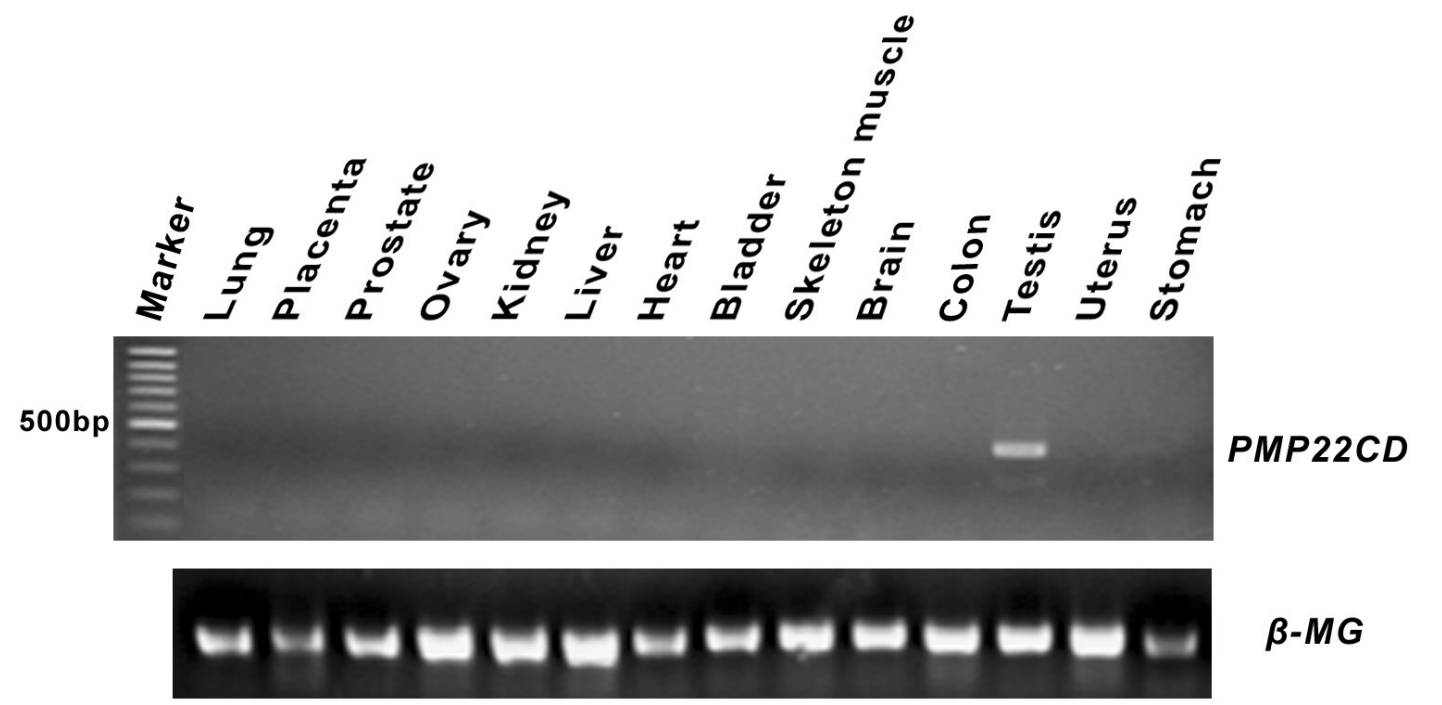

Figure 6. Expression pattern of $P M P 22 C D$ in 14 human tissues. The PCR amplification was performed under these conditions: $94^{\circ} \mathrm{C}$ for 5 min followed by 35 cycles of $20 \mathrm{~s}$ at $94^{\circ} \mathrm{C}, 30 \mathrm{~s}$ at $56.5^{\circ} \mathrm{C}$, 30 s at $72^{\circ} \mathrm{C}$.

\subsection{PMP22CD protein expression and its subcellular localization}

In order to investigate the subcellular location of PMP22CD protein, the PSORT prediction tool was used. The result indicated that PMP22CD was predicted to localize in the cytoplasm, and there was a high possibility of localization in the endoplasmic reticulum. To verify the subcellular location of PMP22CD protein, pEGFP-PMP22CD was transfected into COS7 cells. As shown in Figure 7, when pEGFP-PMP22CD over expressed in COS7 cells, we found that the strongest green fluorescence concentrated around the nuclear membrane, and we also observed granular green fluorescence scattering in the cytoplasm. In our experiment, we did not detect any fluorescence signal in the cell membrane. In contrast, in the cells transfected with pEGFP-C1, the green fluorescence was distributed throughout the whole cell.

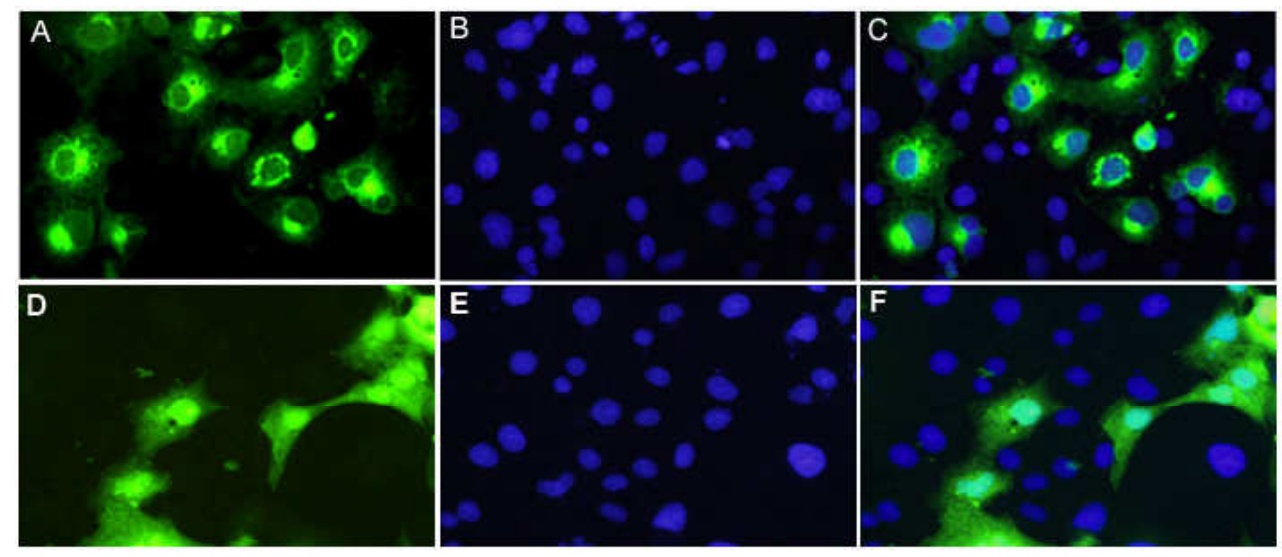

Figure 7. Subcellular localization of PMP22CD protein in COS7 cells.
A: Cells transfected with pEGFP-PMP22CD
$\mathrm{B}$ : The nucleus of cells transfected with pEGFP-PMP22CD were stained with DAPI
C: Colocalization of A and B
D: Cells transfected with pEGFP-C1
E: The nucleus of cells transfected with pEGFP-C1 were stained with DAPI
F: Colocalization of D and E 
To determine the expression and molecular weight of PMP22CD protein, cell lysates transfected with pEGFP-PMP22CD were analyzed by western blotting, and cell lysates transfected with pEGFP$\mathrm{C} 1$ served as a control. The result in Figure 8 showed that the anti-GFP antibody recognized an about $52 \mathrm{kDa}$ protein band of GFP-PMP22CD. As the weight of the GFP-tag is $26 \mathrm{kDa}$, the molecular weight of PMP22CD protein should be around $26 \mathrm{kDa}$, similar to the prediction by Vector NTI.

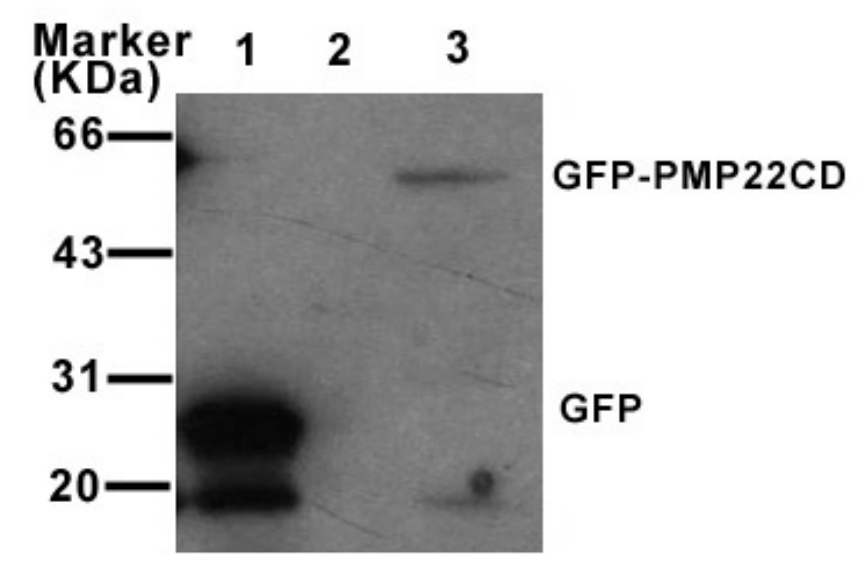

Figure 8. Expression of PMP22CD in COS7 cells. pEGFP-PMP22CD and pEGFP-C1 were transiently transfected into COS7 cells and the cell lysates were analyzed by Western blotting with anti-GFP polyclonal antibody. 1. Cell lysates transfected by pEGFP-C1; 2. Cell lysates not transfected; 3. Cell lysates transfected by pEGFP-PMP22CD.

\section{Discussion}

In the present study, we report the isolation and characterization of a novel human gene $P M P 22 C D$, which is mapped to chromosome 11q24.1. It contains an ORF with a length of $675 \mathrm{bp}$, encoding a protein with a putative PMP22_Claudin domain. PMP22CD protein is composed of four transmembrane helices. The bioinformatics character suggested that PMP22CD is a member of the PMP22/EMP/Claudin family. However, PMP22CD shares low sequence similarity with the other members of PMP22/EMP/Claudin family; it is a distantly related member of this family in evolution.

Previously studies have shown that PMP22/EMP/Claudin family proteins played an important role in cell junctions [3, 4], and that the cell membrane is their main localization site[16-18]. We also determined the localization of PMP22CD in the cell; however, it is not located at the cell membrane. To the contrary, PMP22CD protein is granularly scattered in the cytoplasm and is mainly concentrated around the nuclear membrane. As the outer nuclear membrane is continuous with the membrane system of the endoplasmic reticulum, PMP22CD protein localization is similar to the proteins targeting into the endoplasmic reticulum. The difference of cell localization between PMP22CD and other members of the PMP22/EMP/Claudin family proteins suggests that PMP22CD does not contribute to cell junctions, and that PMP22CD may have a novel function that does not involve cell junctions.

The expression pattern of $P M P 22 C D$ is also different from other claudin family members. Claudin 3 is mainly expressed in the lung and liver[6], claudin 4 is expressed in the kidney[6], claudin 5 is widely expressed in all tissues[6], but highly expressed in the lung, claudin 7 and 8 are both highly expressed in the lung and kidney, claudin 19 is specifically expressed in the kidney[17], and Claudin 1 expression has been detected in testis, lung and brain[19]. Our results indicated that $P M P 22 C D$ is specifically 
expressed in the testis, which is unusual for claudin family members. This was supported by the result of bioinformatics analysis showing that all the ESTs of PMP22CD were transcripted from the testis. The testis is the location of spermatogenesis and sexual hormone production; whether PMP22CD plays a role in regulating these physiological functions remain to be determined.

$P M P 22 C D$ is located at chromosome 11q24.1. A very interesting finding is that twelve olfactory receptor genes(OR6X1, OR6M1, OR6M2P, OR6M3P, OR8D4, OR4D5, OR6T1, OR10S1, OR10G4, OR10G9, OR10G7, OR10G8) are also located at this chromosomal loci, PMP22CD is a fourtransmembrane protein. It is surprising that the olfactory receptor protein is also membrane protein with seven transmembrane helices[20]. Whether PMP22CD and olfactory receptor have a relationship in evolution remains to be investigated. Whether PMP22CD plays a role as a receptor similar to the olfactory receptor in the nuclear membrane remains to be identified in our further studies.

\section{Acknowledgement}

This work was supported by the grant from Start-up Fund for Science Research of Soochow University (Q4134603).

\section{References and Notes}

1. Nagafuchi, A. Molecular architecture of adherens junctions. Curr Opin Cell Biol. 2001, 13, 600603.

2. Furuse, M.; Hirase, T.; Itoh, M.; Nagafuchi, A.; Yonemura, S. and Tsukita, S. Occludin: a novel integral membrane protein localizing at tight junctions. J Cell Biol. 1993, 123, 1777-1788.

3. Muller, H. W. Tetraspan myelin protein PMP22 and demyelinating peripheral neuropathies: new facts and hypotheses. Glia. 2000, 29, 182-185.

4. Furuse, M.; Fujita, K.; Hiiragi, T.; Fujimoto, K. and Tsukita, S. Claudin-1 and -2: novel integral membrane proteins localizing at tight junctions with no sequence similarity to occludin. $J$ Cell Biol. 1998, 141, 1539-1550.

5. Tsukita, S. and Furuse, M. Overcoming barriers in the study of tight junction functions: from occludin to claudin. Genes Cells. 1998, 3, 569-573.

6. Morita, K.; Furuse, M.; Fujimoto, K. and Tsukita, S. Claudin multigene family encoding fourtransmembrane domain protein components of tight junction strands. Proc Natl Acad Sci U S A. 1999, 96, 511-516.

7. Tsukita, S.; Furuse, M. and Itoh, M. Multifunctional strands in tight junctions. Nat Rev Mol Cell Biol. 2001, 2, 285-293.

8. Christophe-Hobertus, C.; Szpirer, C.; Guyon, R. and Christophe, D. Identification of the gene encoding Brain Cell Membrane Protein 1 (BCMP1), a putative four-transmembrane protein distantly related to the Peripheral Myelin Protein 22 / Epithelial Membrane Proteins and the Claudins. BMC Genomics. 2001, $2,3$.

9. Kearsey, J.; Petit, S.; De Oliveira, C. and Schweighoffer, F. A novel four transmembrane spanning protein, CLP24. A hypoxically regulated cell junction protein. Eur J Biochem. 2004, 271, 25842592. 
10. Gonzalez-Mariscal, L.; Betanzos, A.; Nava, P. and Jaramillo, B. E. Tight junction proteins. Prog Biophys Mol Biol. 2003, 81, 1-44.

11. Huang, C.; Guo, J.; Liu, S.; Shan, Y.; Wu, S.; Cai, Y. and Yu, L. Isolation, tissue distribution and prokaryotic expression of a novel human X-linked gene LHFPL1. DNA Seq. 2004, 15, 299-302.

12. Huang, C.; Zhou, J.; Wu, S.; Shan, Y.; Teng, S. and Yu, L. Cloning and tissue distribution of the human B3GALT7 gene, a member of the beta1,3-Glycosyltransferase family. Glycoconj J. 2004, 21, 267-273.

13. Altschul, S. F.; Madden, T. L.; Schaffer, A. A.; Zhang, J.; Zhang, Z.; Miller, W. and Lipman, D. J. Gapped BLAST and PSI-BLAST: a new generation of protein database search programs. Nucleic Acids Res. 1997, 25, 3389-3402.

14. Tusnady, G. E. and Simon, I. The HMMTOP transmembrane topology prediction server. Bioinformatics. 2001, 17, 849-850.

15. Wang, M.; Wang, J.; Zhang, Z.; Zhao, Z.; Zhang, R.; Hu, X.; Tan, T.; Luo, S. and Luo, Z. Dissecting phenotypic variation among AIS patients. Biochem Biophys Res Commun. 2005, 335, 335-342.

16. Inai, T.; Sengoku, A.; Guan, X.; Hirose, E.; Iida, H. and Shibata, Y. Heterogeneity in expression and subcellular localization of tight junction proteins, claudin-10 and -15 , examined by RT-PCR and immunofluorescence microscopy. Arch Histol Cytol. 2005, 68, 349-360.

17. Lee, N. P.; Tong, M. K.; Leung, P. P.; Chan, V. W.; Leung, S.; Tam, P. C.; Chan, K. W.; Lee, K. F.; Yeung, W. S. and Luk, J. M. Kidney claudin-19: localization in distal tubules and collecting ducts and dysregulation in polycystic renal disease. FEBS Lett. 2006, 580, 923-931.

18. Li, W. Y.; Huey, C. L. and Yu, A. S. Expression of claudin-7 and -8 along the mouse nephron. Am J Physiol Renal Physiol. 2004, 286, F1063-1071.

19. Gye, M. C. Expression of claudin-1 in mouse testis. Arch Androl. 2003, 49, 271-279.

20. Sengupta, P.; Chou, J. H. and Bargmann, C. I. odr-10 encodes a seven transmembrane domain olfactory receptor required for responses to the odorant diacetyl. Cell. 1996, 84, 899-909.

Note: The sequence data reported here have been submitted to the GenBank database under accession number AY634366.

(C) 2006 by MDPI (http://www.mdpi.org). Reproduction is permitted for noncommercial purposes. 